\title{
Visual Effects of Lesions of Cortical Area V2 in Macaques
}

\author{
William H. Merigan, Tara A. Nealey, ${ }^{a}$ and John H. R. Maunsell ${ }^{b}$ \\ Center for Visual Science, University of Rochester, Rochester, New York 14642
}

Ibotenic acid lesions were placed in two monkeys in a portion of cortical area V2 that corresponds to a lower quadrant of the visual field extending approximately $3-7^{\circ}$ from the fovea. For purposes of comparison, another lesion was placed in area $\mathbf{V} 1$ in one animal. $A$ wide range of visual capacities were then measured, using a discrimination between vertical and horizontal orientation, in and near the affected regions of the visual field. Visual acuity declined sharply as the test stimulus approached the visual field location corresponding to the $V_{1}$ lesion, and no threshold could be measured at its center. In contrast, lesions of area V2 caused no measurable decrease in acuity, nor was there any substantial effect on several measures of contrast sensitivity.

Subsequently, two types of more complex visual discriminations were measured (also using a vertical-horizontal discrimination), and these discriminations were severely disrupted by $\mathrm{V} 2$ lesions. The first discrimination was of the orientation of two parallel lines of five colinear dots each. We measured the number of background dots that would bring the discrimination to threshold, and this number of dots was greatly decreased by a V2 lesion. The second discrimination was of the orientation of a group of three distinctive texture elements embedded in a six by six element texture. This task could not be done in the visual field region affected by the $\mathbf{V} 2$ lesion when the distinctive elements differed in orientation from the others. Control experiments showed that the discrimination could be done when the three distinctive elements differed in size or color. These results suggest that cortical area $\mathbf{V} 2$ is not needed for some low-level discriminations, but may be essential for tasks involving complex spatial discriminations.

IKey words: extrastriate, visual cortex, contrast sensitivity, orientation discrimination, parallel pathways, macaque monkey]

Cortical area $\mathrm{V} 2$, which is now known to contain at least three subregions, lies immediately adjacent to, and receives much of its input from, cortical area V1 (striate cortex). Area V1 is the conduit for virtually all geniculocortical projections, and lesions

\footnotetext{
Received Oct. 30, 1992; revised Jan. 20, 1993; accepted Feb. 11, 1993.

We thank James Jester for assistance in testing monkeys and Peter Vamvakius for histology and lesion reconstruction. Dr. Tatiana Pasternak commented on the manuscript. This research was supported by Grants ES01247, EY01319, EY05911, and EY08898.

Correspondence should be addressed to William H. Merigan, Box 314, University of Rochester Medical Center, Rochester, NY 14642.

${ }^{2}$ Present address: Neurosurgery Research Laboratory, Massachusetts General Hospital, Boston, MA 02114.

b Present address: Division of Neurosciences, Baylor College of Medicine, Houston, TX 77030.

Copyright (c) 1993 Society for Neuroscience $0270-6474 / 93 / 133180-12 \$ 05.00 / 0$
}

of area V1 in macaque or human usually result in almost complete blindness (Koerner and Teuber, 1973; Miller et al., 1980). Area V2, on the other hand, operates in parallel with other pathways to extrastriate cortex (Van Essen, 1985), and it is not known how severely its removal affects vision. While lesions have been made in prestriate areas of macaque visual cortex (e.g., Denny-Brown Chambers, 1976), it is unclear which portions of V 2 were damaged by these lesions, and testing was not done with controlled visual fixation. In humans, damage to the likely counterpart of macaque area V2 (Clarke and Miklossy, 1990) results in markedly different effects depending on whether the lesion is dorsal or ventral to the calcarine fissure (Damasio and Damasio, 1989). This qualitative difference may be due to damage extending to underlying white matter, thus affecting striate input to a wide region of extrastriate cortex. These observations suggest that there may be no counterpart in human clinical cases to the lesions of the present study, which were confined to neurons and spared fiber tracts. For example, lesions near human V2 that result in field loss in the lower visual field, with a sharp border along the horizontal meridian (Horton and Hoyt, 1991b), have been interpreted as due to lesions of cortical area $\mathrm{V} 2$ or $\mathrm{V} 2$ and $\mathrm{V} 3$, but may in fact reflect damage to fiber tracts.

That cortical areas V1 and V2 might make different contributions to visual processing is not strongly suggested by the physiological properties of their neurons. The two areas show great similarities in selectivity for such stimulus features as orientation, direction, color, contrast, and so on. However, there are differences. Neurons in area V2 have larger receptive fields and are responsive to somewhat lower spatial frequencies (Foster et al., 1985) than those in V1. There also appear to be somewhat more binocularly driven cells in V2 than V1 (Poggio et al., 1988). In addition, recent reports have described a dramatic difference in the response of V1 and V2 neurons to such complex stimulus features as illusory contours (Heydt and Peterhans, 1989), but these findings remain controversial (Grosof et al., 1992).

The goal of the present study was to determine what changes in visual performance result from damage to area V2. Because $\mathrm{V} 2$ is the target for much of the neuronal output of area V1, we first compared the effects of $\mathrm{V} 1$ and $\mathrm{V} 2$ lesions on basic visual capacities. V1 lesions devastated vision, but since we found little change in basic visual capacities after V2 lesions, we next examined effects of these lesions on visual discriminations that require more complex processing. The two tasks used in these experiments involved the recognition of colinearity of dots, and the discrimination of shape from local orientation, discriminations that were designed to test the grouping of local features. These tasks were suggested in part by single-unit studies of V1 and V2 neurons (Heydt and Peterhans, 1989; Grosof et al., 1992) 
and by modeling studies of cortical processing (Sutter et al., 1989; Malik and Perona, 1990). Since the results suggested that such discriminations could not be done in the absence of V2, three control experiments were run to rule out alternative accounts of the $\mathrm{V} 2$ related deficits.

\section{Materials and Methods}

All experiments were carried out in accordance with U.S. Public Health Service guidelines (NIH Guide for the Care and Use of Laboratory Animals, 1978).

\section{Subjects}

The subjects were two adult female monkeys (Macaca nemestrina) of approximately $5 \mathrm{~kg}$ body weight. They had free access to monkey chow, supplemented regularly with fresh fruit, and their water was withheld for approximately $20 \mathrm{hr}$ before threshold testing $5 \mathrm{~d}$ each week. All testing was done binocularly using controlled fixation.

\section{Placement of lesions}

Lesions of area V2 were made along an $8 \mathrm{~mm}$ portion of the posterior bank of the dorsal lunate sulcus extending from about 8 to $16 \mathrm{~mm}$ from the midline. In an aseptic procedure the cortical surface was exposed with a craniotomy and durotomy, and a $10 \mathrm{~mm}$ stretch of the posterior bank of the lunate sulcus was mapped with microelectrodes. When mapping was complete, the electrode was replaced with a recordinginjecting probe (Schiller and Malpeli, 1977), and a grid of injections of ibotenic acid. $(10 \mu \mathrm{g} / \mu \mathrm{l})$ was made with approximately $1 \mathrm{~mm}$ spacing. Forty-one injections of $750 \mathrm{nl}$ were made in left V2 of monkey 857 (lower right visual field), 51 such injections in left V2 of monkey 9102 (lower right visual field), and 41 injections of $1.0 \mu \mathrm{l}$ in right $\mathrm{V} 2$ of monkey 9102 (lower left visual field). In addition, a lesion was made in ventral V1 of the right hemisphere of monkey 9102 (upper left visual field), by placing 50 injections of $750 \mathrm{nl}$ in a square $6 \times 6 \mathrm{~mm}$. Individual injections were spaced $1 \mathrm{~mm}$ center to center and were about $1.5 \mathrm{~mm}$ below the cortical surface.

The injections in the left hemisphere of monkey 9102 produced no visual effects (tested with all of the tasks described in this article), and there was no sign of any morphological changes post-mortem. We do not know why these injections failed to produce a lesion.

\section{Implantation of scleral search coil and headmount}

In separate procedures, after placement of the lesion in monkey 857 and before placement of the first lesion in 9102 , a scleral search coil was implanted in the right eye of each monkey, under isoflurane anesthesia, so that eye position could be monitored. At the same time a stainless steel sleeve was attached to the skull so that the monkey's head could be immobilized.

\section{Sequence of lesions and testing}

In monkey 9102, the left V2 lesion was attempted (unsuccessfully; see above), followed 5 months later by the V1 lesion, and then 2 months after that by the successful right V2 lesion. This monkey was killed 10 months after the V1 lesion and 8 months after the V2 lesion. In monkey 857 , the single V2 lesion was followed 18 months later by death. After each of the lesions, visual acuity was mapped throughout the visual field for approximately 2 months, and then contrast sensitivity was measured for approximately an additional month. The remaining more complex discriminations were then tested in isolation for another 3 months, before each of these functions was rechecked to determine if there had been any long-term changes.

\section{Apparatus and procedures}

\section{Visual acuity}

Acuity was tested as previously described (Merigan et al., 1991a), except that a stimulus size of $1.6^{\circ}$ was used for measurements. Briefly, the monkey fixated a laser spot placed so as to arrange the correct retinal locus of a $1.6^{\circ}$ diameter circular patch of sinusoidal grating displayed on a high-resolution monitor ( $\mathrm{P}-31$ phosphor, 0.55 Michaelson contrast, $16 \mathrm{~cd} / \mathrm{m}^{2}$ ). The monkey responded by pressing on a left or right pushbutton depending upon whether the grating was horizontal or vertical. Grating spatial frequency was adjusted according to a staircase proce-

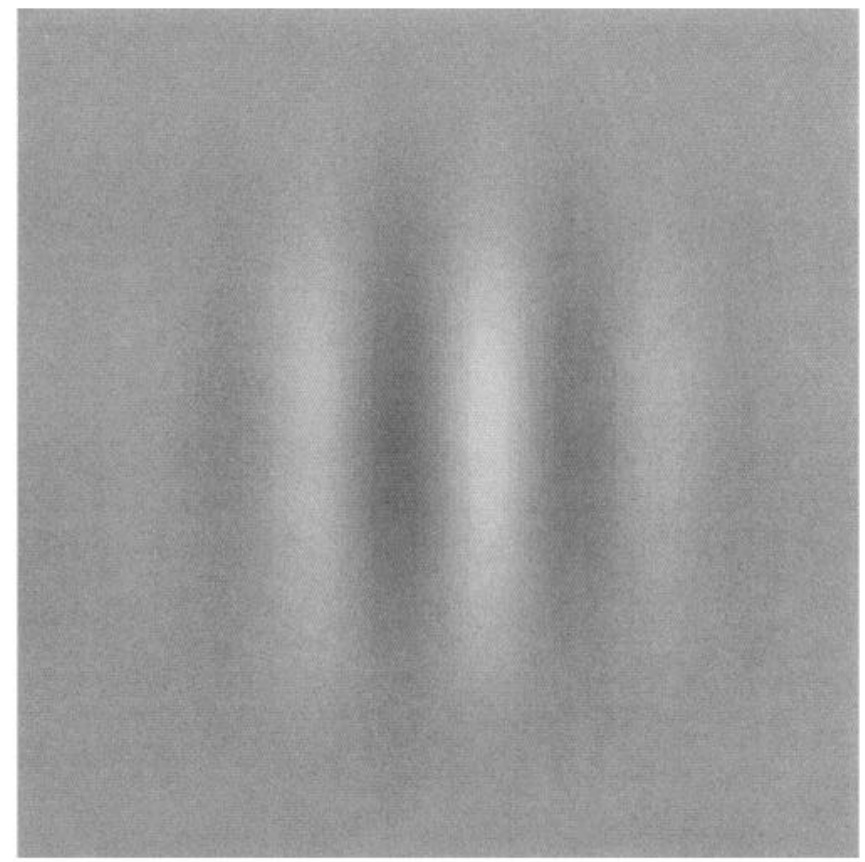

Figure 1. Picture of the type of localized grating patch (Gabor stimulus) used to test contrast sensitivity. The stimulus consists of a vertical or horizontal cosinusoidal grating multiplied by a vertical and horizontal Gaussian envelope. Such a stimulus is relatively localized in both space (location on the retina) and spatial frequency (frequency of the cosinusoidal gratting). For testing chromatic sensitivity, the cosinusoidal grating varied in color, not in luminance as shown here. When testing sensitivity to drifting stimuli, the Gaussian envelope remained in place, and the cosinusoidal grating drifted to the right.

dure in 0.3 octave steps, and threshold was taken at $75 \%$ correct from the daily psychometric function. In this and all the other procedures described below, test sessions lasted 200 trials. In addition, trials were terminated (and stimuli turned off) when fixation deviated more than $0.3^{\circ}$ from the fixation spot, and data from terminated trials were discarded.

\section{Contrast sensitivity}

Testing procedures have been described in detail (Merigan et al., 1991a,b). The monkey fixated a laser spot projected on the face of a 19 inch color monitor (Conrac 7211) placed at a distance of $211 \mathrm{~cm}$. Contrast thresholds were measured with small patches of grating (Gabor functions) (Fig. 1) generated on an Adage 3006 raster display unit and presented at a frame rate of $60 \mathrm{~Hz}$. The horizontal and vertical Gaussian weighting functions had space constants $(s)$ of $1.14^{\circ}$, so that the grating was above $37 \%$ of peak contrast (full width at the $1 / e$ point), over a region of $2.28^{\circ}$. Once the monkey fixated, stimuli were presented with a slow and gradual onset (contrast was raised from 0 to full contrast according to one-half cycle of a raised cosine of $0.5 \mathrm{~Hz}$, i.e., slowly came on over $1 \mathrm{sec}$ ), and stimuli remained on until either a fixation break or a response. The contrast of the stimulus was varied from trial to trial according to a staircase, becoming easier by one step ( $2 \mathrm{~dB}$ contrast) after each error, and harder, with probability 0.33 , after each correct choice. Daily sessions consisted of 200 trials, and thresholds were taken at $75 \%$ correct responding either by linear interpolation or by probit fits to the daily psychometric functions (Finney, 1971).

Luminance contrast sensitivity for stationary gratings. The cosinusoidal component of the Gabor stimulus was a 1 cycle/degree luminance grating. These gratings were presented in either horizontal or vertical orientation, and the monkey indicated the orientation by pressing a left or right pushbutton.

Chromatic contrast sensitivity for stationary gratings. The cosinusoidal component of the stimulus was a 1 cycle/degree isoluminant chromatic grating that was modulated in a red-green (i.e., constant blue) direction in the MacLeod-Boynton color space (Derrington et al., 1984; Merigan, 1989). Chromatic contrast sensitivity was taken as the sum 


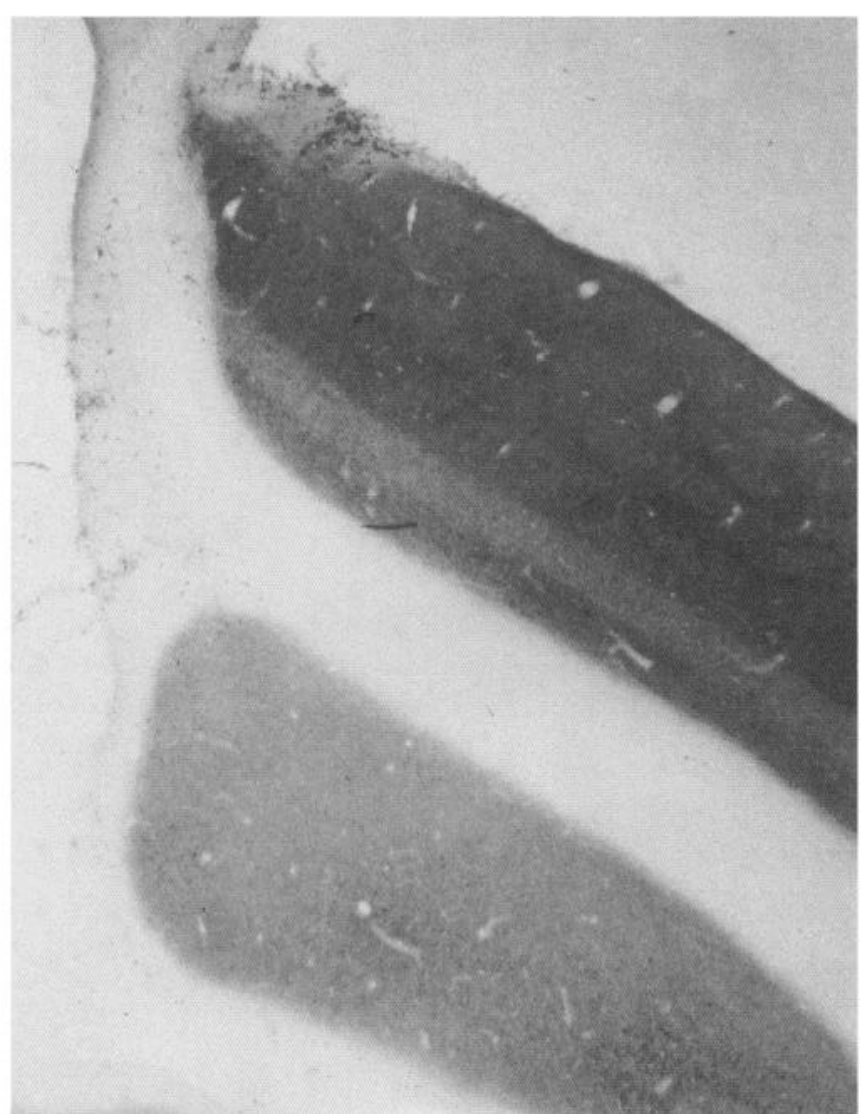

Figure 2. Parasagittal frozen section from the lateral portion of occipital cortex of monkey 857, showing the appearance of the V2 lesion. The section was stained to demonstrate cytochrome oxidase. In this figure dorsal is up and anterior is to the left.

of the modulations of the middle- and long-wavelength cones. In addition, the tritanopic direction in color space (approximately yellowblue) was also tested for monkey 857 . As above, sensitivity was tested by having the monkey discriminate grating orientation.

Contrast sensitivity for the detection of drifting gratings. The cosinusoidal component of the stimulus was a 1 cycle/degree luminance grating drifting at 10 degrees $/ \mathrm{sec}(10 \mathrm{~Hz})$. A yes-no procedure was used to test contrast thresholds. On each "yes" trial (presented randomly on half the trials) a vertical Gabor stimulus was presented during fixation, and the Gaussian envelope remained fixed while the cosinusoidal component drifted to the right. The correct response was to the right pushbutton on such trials. On "no" trials, no stimulus was presented, and the correct response was a press on the left pushbutton.

Contrast sensitivity for discriminating the direction of motion. Stimuli were the same as in the previous experiment except that a Gabor stimulus was presented on every trial and the cosinusoidal component drifted either to the right or to the left. The right pushbutton was used to signal rightward motion, and the left to signal leftward motion.

\section{Complex discriminations}

Colinearity of groups of dots. Stimuli like those shown at the top of Figure 9 were presented, one on each trial. One group of stimuli contained two lines of five horizontally aligned dots like that shown at the top left of Figure 9, and for such stimuli a press on the left pushbutton was correct. The other group of stimuli contained two lines of vertically aligned dots like those shown in the top right of Figure 9, and with such stimuli the right pushbutton was correct. On each trial the number of background masking dots was controlled by a staircase with one fewer masking dot presented after each error, and one more (with probability 0.33 ) after each correct response. The location of each masking dot had a fixed centroid and a random horizontal and vertical displacement of up to one-fourth the separation of the lines of dots. The stimuli shown in Figure 9 have seven masking dots. These stimuli were presented as black squares on a white monitor screen and the full stimulus subtended $2 \times 2$ degrees of visual angle.

Discriminating the orientation of triads of texture elements. Stimuli for the first experiment are illustrated in the top left and right of Figure 10 . On roughly half of the trials a stimulus like that shown in the top left of Figure 10 was presented, with the three right oblique line segments forming a horizontal group. This group always was shown within a central region consisting of the middle four columns and middle two rows. On such trials a left pushbutton response was correct. On the other trials a stimulus like that shown in the right part of Figure 10 was shown. Again the location of the stimulus was confined to a central region consisting of the central two columns and central four rows. On such trials a right response was correct. These stimuli were presented as black lines on a white monitor and the full stimulus subtended $2 \times 2$ degrees of visual angle.

Detection of single misoriented texture element. As shown in Figure 11 , the texture presented on each trial sometimes contained a single right oblique line segment. On trials with no misoriented line segment (left of Fig. 11), left pushbutton responses were correct, and on the other trials (right of Fig. 11), right responses were correct.

Discriminating the orientation of triads of thicker line elements. As shown in Figure 12, this task was identical to that illustrated above in Figure 10, except that the three misoriented line segments were made three times as thick. This provided a luminance cue to the orientation of the group that was not present in the earlier task.

Discriminating the orientation of triads of square patches of color. For this experiment the small line segments of the texture were replaced by small square patches of color on a white display. Figure 13 shows the arrangement of the task, although the colors are represented here by different patterns. The two colors used were reddish and greenish, matching the colors used above in chromatic gratings, and fell along a constant-blue axis in opponent color space (Merigan, 1989). After initial testing with these stimuli, the relative intensity of the reddish and greenish stimuli was varied over sessions to establish that this task could also be done at isoluminance. As above, the stimuli subtended $2 \times 2$ degrees of visual angle.

\section{Reconstruction of lesions}

At the conclusion of behavioral testing, the lesions placed in both monkeys were reconstructed with physiological and anatomical techniques. Immediately prior to death, multiunit physiological recordings were used to map the portion of cortical areas V1 and V2 surrounding the lesion. Small electrolytic lesions $(10 \mu \mathrm{A} \times 10 \mathrm{sec})$ were made to facilitate reconstruction of the penetrations. After physiological recording, the monkey was killed and perfused with a saline rinse followed by $4 \%$ paraformaldehyde in phosphate-buffered saline. The brain was removed and blocked, and $40 \mu \mathrm{m}$ sections were cut on a freezing microtome. One section in eight was reacted for cytochrome oxidase activity (WongRiley, 1979), and another stained for Nissl substance. The cytochrome oxidase stain provided a clear indication of cortical regions affected by the ibotenic acid, and the Nissl sections were used to assess more precisely the limits of regions without surviving neurons. These sections were used to reconstruct the extent of the lesions and their relationship to physiological recording sites.

\section{Results}

\section{Lesion reconstructions}

Figure 2 shows an example of a section from which the V2 lesions were reconstructed. The section was cut in a parasagittal plane and stained for cytochrome oxidase. The top portion of the section shows intact V1 on the opercular surface of the brain. The lesion begins just posterior to the V1/V2 border, which itself was just posterior to the lunate sulcus in cortex, and which corresponds to the vertical meridian of the visual field. The lesion extends approximately $2.5 \mathrm{~mm}$ down the posterior bank of the lunate sulcus.

Lesions were successfully placed in V2 of monkey 856, and in V1 and V2 in the right hemisphere of monkey 9102 . No histological evidence was found of any damage corresponding the attempted V2 lesion in the left hemisphere of monkey 9102, nor was there any behavioral evidence for visual loss in the 
corresponding hemifield of that animal. We do not know why the injections failed to produce a lesion in this case.

2-D flattened maps of cortex (Van Essen and Maunsell, 1980) were constructed for the successfully ablated hemispheres using histological sections in conjunction with the reconstruction of recording site locations. Maps of V1 and adjacent extrastriate cortex from these hemispheres are shown in Figure 3. Each map illustrates visual cortex as if it had been peeled away from white matter and then unfolded and laid flat. The elliptical region to the left is the entirety of V1. Parts of V2, V3, and other extrastriate visual areas lie in the large region to the right. Dotted lines mark the fundi of the sulci that have been opened. The fundi of the calcarine sulcus and the lunate sulcus have been labeled. Regions of cortex in which injections of ibotenic acid caused complete lesions are marked in black, and those with partial lesions are marked by stippling.

The lesion in monkey 857 was centered in V2, and spread only a short distance into adjacent V1. The lesion appears discontinuous in the map in Figure $3 A$ because a split was made at the $\mathrm{V} 1 / \mathrm{V} 2$ border to reduce distortions resulting from the flattening. Lesioned cortex extended well beyond the fundus of the lunate sulcus, encroaching on V3. Thus, the lesion spanned a segement of the lower field representation in V2 from the vertical meridian to the horizontal meridian. A corresponding representation was ablated in monkey 9102, but with more collateral damage. In this animal the ibotenic acid spread through the white matter dorsal and posterior to V2, and extensively damaged overlying portions of $\mathrm{Vl}$ in the operculum. This is visible as the large region extending down from the upper border of the $\mathrm{V} 1$ map in Figure $3 B$. Ibotenic acid from the $\mathrm{V} 2$ injections also spread ventrally to damage a small stretch of $\mathrm{V} 1$ cortex in the calcarine sulcus (upper left V1 lesion in Fig. $3 B$ ). In monkey 9102 we intentionally made a lesion of the upper field representation in V1. This lesion appears in the lower right section of the $\mathrm{V} 1 \mathrm{map}$ in Figure $3 B$. Ibotenic acid from this lesion spread through the white matter and into the calcarine sulcus, producing the small lesion in the lower left part of the map. The accidental lesions in the calcarine sulcus of monkey 9102 affected visual field eccentricitries beyond those tested behaviorally, and will not be considered further.

The relationship of the lesions to visual field representations in V1 and V2 is shown in Figure 4. Separate axes plot visual field locations corresponding to V1 (above) and V2 (below). The receptive fields that were plotted during the final mapping are marked with dots in Figure 4 . The borders of the lesions were located in a series of histological sections and related to visual field loci using nearby recording sites. Although portions of the lower field representation were damaged in both V1 and V2, there were regions of the quadrant for which $\mathrm{V} 2$ was involved and Vl was not. In these regions it was possible to assess the effects of a purely extrastriate lesion.

\section{Visual testing}

Latency from trial (and stimulus) onset to response in the two monkeys ranged from approximately $150-500 \mathrm{msec}$ for all of the tasks described below. Latencies did not appear to differ from task to task, but rather to grow progressively shorter from day to day as a single task was repeated. There was no apparent change in latency from before to after placement of the lesions.

Figure 5 shows visual acuity over the upper visual field of monkey 9102 and the reconstructed location of the V1 lesion in this monkey. No alteration was seen in measured acuity
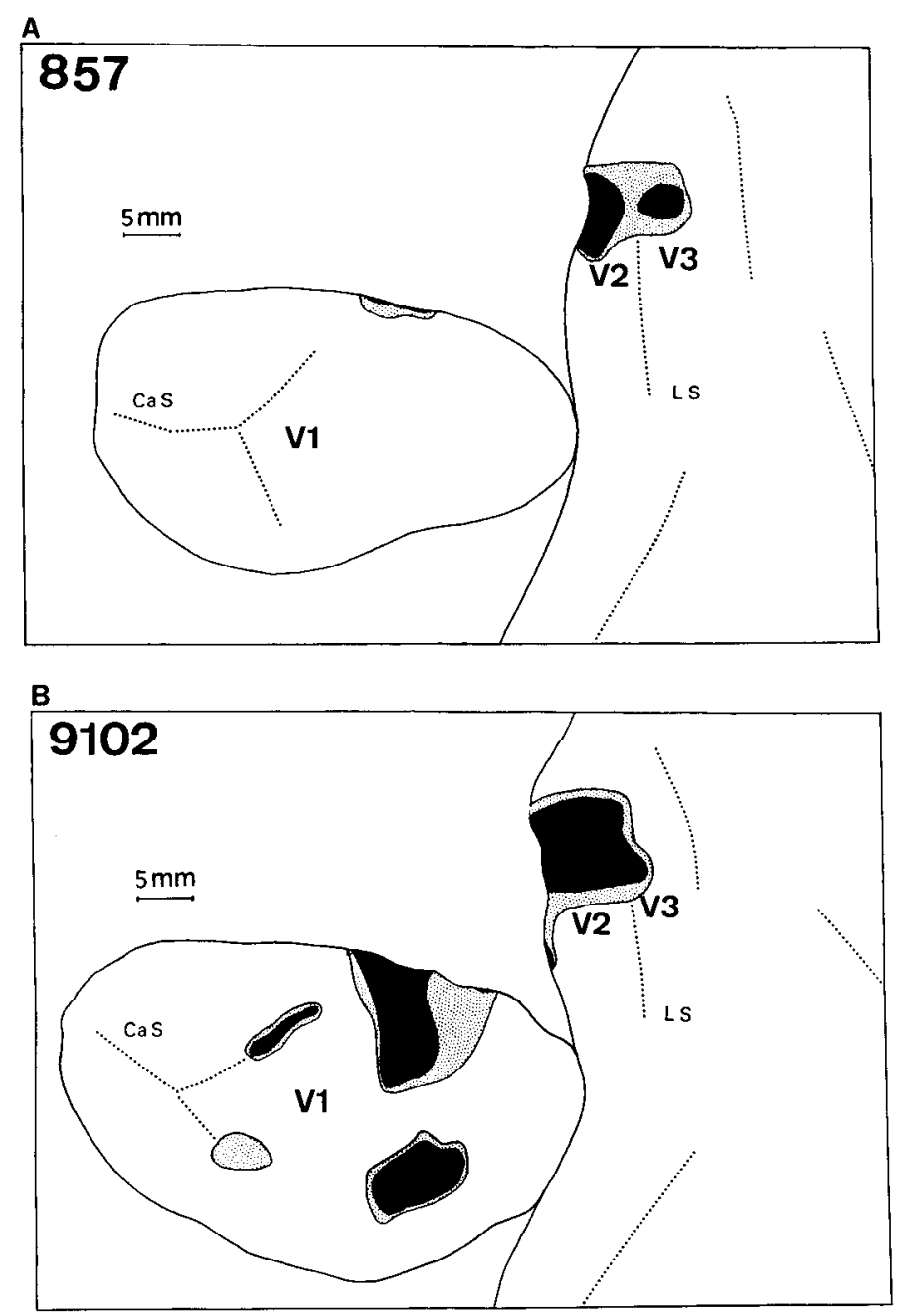

Figure 3. Reconstruction of lesions on a flattened representation of visual cortex (Van Essen and Maunsell, 1980). This approach allows 3-D information about cortex obtained from histological sections to be displayed on a 2-D map. The borders of area V1 actually contact the borders of V2 (vertical contour to the right of center), but geometrical constraints dictate that they be shown as separated. Dotted lines show the location of the deepest points (fundi) of sulci, and two of these, the calcarine $(\mathrm{CaS})$ and lunate $(L S)$, are labeled. Black areas represent regions of complete damage to cortex, and stippled areas, partial damage.

throughout the course of postlesion testing. However, during the first week of testing after the lesion, the monkey would not respond when tested within $2^{\circ}$ of the apparent center of the scotoma ( $2^{\circ}$ up and $4.5^{\circ} \mathrm{left}$ ), and simply broke fixation repeatedly. On intervening tests at other locations acuity was comparable to prelesion values. After the first week of testing, the monkey began to respond at all locations, and initial measures were comparable to those measured later. No acuity threshold could be measured, although the monkey responded well, within the central portion of the Vl lesion, and the spatial transition from no measurable acuity to normal acuity took place over less than $1^{\circ}$. These results likely underestimate the size of the lesion since the test stimulus was large $\left(1.6^{\circ}\right.$ diameter $)$ and much of the stimulus probably had to fall within the affected area before loss was evident. The extent of the region of no measurable acuity was about $1.5^{\circ}$, and within this region we were also unable to measure even simple detection of a stationary or rapidly drifting grating stimulus (contrast 0.55 , I cycle/degree, drifting at 0-10 degrees/sec). 
A 857
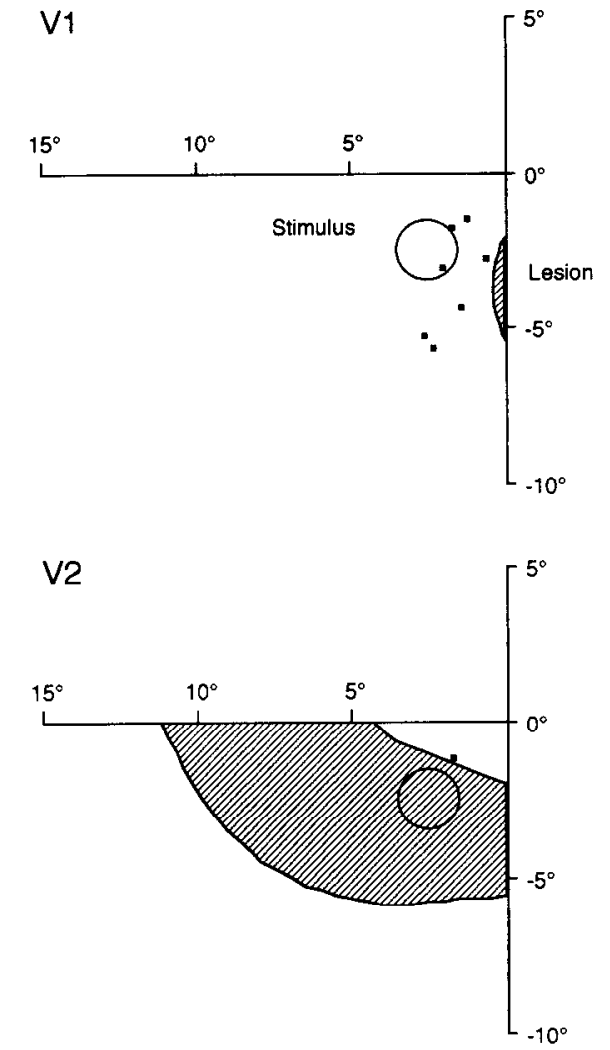

B 9102
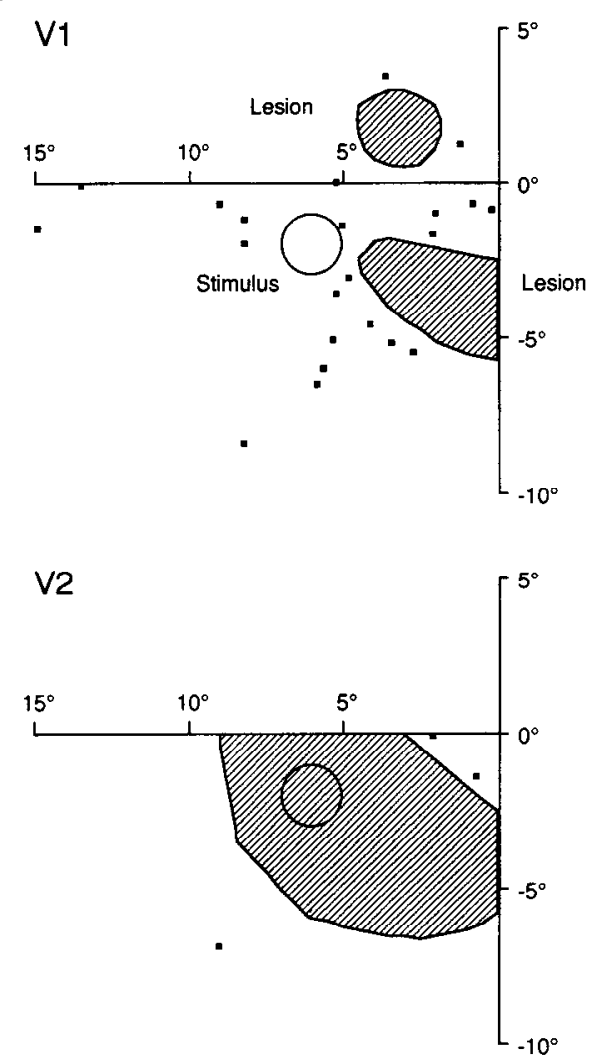

A different view of the acuity loss in the region of the lesion can be seen in Figure 6, which shows the visual acuity of monkey 9102 along a horizontal plane through the visual field at $2^{\circ}$ above the horizontal meridian. It can be seen in this figure that acuity loss was complete within the region of the V1 lesion.

Figure 7 shows visual acuity over the lower visual fields of monkeys 857 and 9102 , and the location of the V2 lesions in these fields (dashed contours), as well as the location of the inadvertent damage to area V1 (dotted contours). Circles show the visual field location of stimuli used in the subsequent studies described below. We found an approximately threefold loss of acuity within the V2 lesion for monkey 857 for the first 3 weeks of tcsting. Mcasured acuity then abruptly jumped to the normal levels shown in this figure. No evidence of improvement over time was seen for monkey 9102 , although this monkey did show a stable disruption of visual acuity that was especially severe at about $2^{\circ}$ eccentricity near the vertical meridian, and extended across a large portion of the visual field. Reconstruction of the lesion in this monkey indicated that the areas of most marked acuity loss corresponded to unintended damage to cortical area $\mathrm{V} 1$. In the regions shown by the circle in the lesion hemifield we found no damage to area V1, complete destruction of area V2, and no evidence of acuity loss. All of the testing described below was done at this location.

Figure 8 shows four measures of contrast sensitivity for monkeys 9102 and 857 in the V2 lesion. Luminance contrast sensitivity, measured with stationary gratings $(0 \mathrm{~Hz})$, was not significantly altered in either monkey. Red-green chromatic contrast sensitivity, measurcd with the same spatiotemporal frequencies, was reduced about twofold in monkey 857 , but not reduced in monkey 9102 . No change in tritanopic contrast sensitivity was found for monkey 857 . Contrast sensitivity was also measured for detection and direction discrimination with $10 \mathrm{~Hz}$ drifting gratings in both monkeys, and no loss was found.

Thus, to this point we had found devastating long-term loss of all tested visual functions after a V1 lesion, but no substantial change in acuity or contrast sensitivity after V2 lesions. The remainder of the tests described below examined whether more complex visual functions would be disrupted by the V2 lesion.

The upper part of Figure 9 shows examples of the stimuli used to measure orientation discrimination for lines of five colinear dots against a background of irregularly placed dots. The left stimulus shows horizontal lines of dots on a background of seven dots, and the right stimulus shows vertical lines of dots on a background of seven dots. Control performance for the two monkeys indicates that this discrimination could be made in the presence of backgrounds of up to 10-15 dots. However, in the visual field corresponding to a V2 lesion, these thresholds were reduced by about a factor of three, a result that was significant for 857 but was too variable to be significant for 9102 . The approximately threefold reduction for 9102 was confirmed

Figure 4. Projection of the cortical lesions onto maps of the visual field, one for damage to area V1 (above) and a second for area V2 (below). Vertical and horizontal axes represent the vertical and horizontal meridia of the visual field. Small squares show the locations of receptive fields mapped during the final recording scssion, which were used to relate cortical loci to locations in the visual field. The circles in the lower field maps show the location of test stimuli used to examine the effect of V2 lesions on contrast sensitivity, as well as on the orientation of lines of dots and texture elements. 


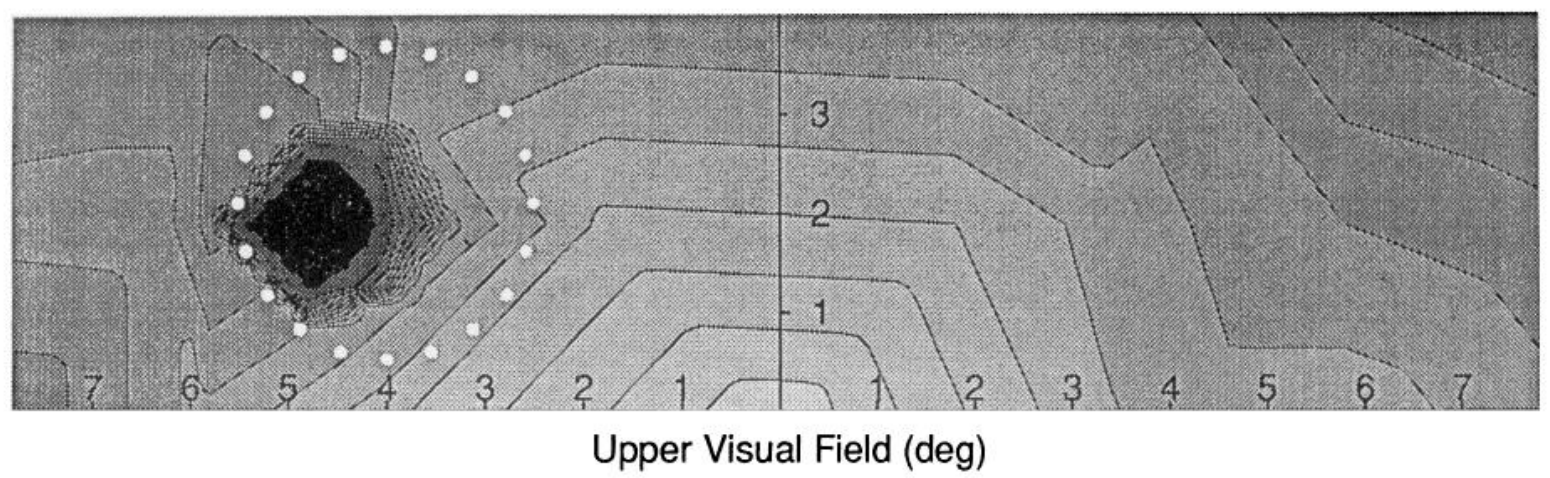

Figure 5. Visual acuity across the upper visual field of monkey 9102 . Visual acuity was measured at a $2^{\circ}$ spacing vertically and horizontally across the visual field, except around the lesion where samples were taken at $0.5^{\circ}$ spacing. The surface, fit by interpolation of cubic splines to these points, passes through all data points. Highest acuity was at the fovea ( $0^{\circ}$ on horizontal and vertical meridia), and each contour represents a decrease of approximately $10 \%$ of that value. The circle of white dots represents the location of the V1 lesion calculated from physiological and histological mapping of the lesion.

at two other visual field locations at which there were V2 (but not V1) lesions, $6^{\circ}$ below and $4^{\circ}$ left, and $4^{\circ}$ below and $6^{\circ}$ left of fixation.

Percent correct performance in discriminating the orientation of distinctive line segments is shown in Figure 10. Both monkeys performed over $85 \%$ correct in the control portion of the visual field. However, neither monkey was able to perform reliably above chance $(50 \%)$ in the region corresponding to the $\mathrm{V} 2$ lesion. We are confident that this lack of discrimination ability was not due simply to lack of familiarity with the task. Both monkeys showed discrimination performance of over $80 \%$ within the first two sessions in the control part of the visual field, and showed no difficulty with the task for all field loci tested in this quadrant, which included all locations out to $6^{\circ}$ eccentricity. However, within the V2 lesion they failed to reach high levels of performance despite over 20 test sessions in different regions of the visual field within the lesion quadrant.

The next three figures show performance of the same monkeys on tasks designed to clarify the basis of the disrupted performance on the discrimination shown in Figure 10. In the first task (Fig. 11), the monkey reported whether or not a single right oblique line segment was present. With this task, the performance of both monkeys within the lesion rapidly improved to over $80 \%$. In a second variant of the original discrimination (Fig. 12), the three unique line segments were made to differ in width as well as orientation from the surrounding segments. This difference greatly improved the performance of monkey 857 , raising lesion locus performance to approximately $80 \%$, while that of monkey 9102 also improved, but only to about $75 \%$. In a final control procedure (Fig. 13), the textures were made up of small squares of color rather than oriented line segments. Again, the monkeys were able to perform this task at levels substantially above chance, and this high level of performance was not affected when the relative luminance of the two colors was varied around isoluminance.

\section{Discussion}

In this first controlled fixation study of the visual effects of V2 (as well as partial V3) lesions, we found no alteration in visual acuity and little or no change in contrast sensitivity. Conversely, a small lesion of area V1 caused an apparently complete loss of visual function in the corresponding region of the visual field, with no recovery over the 10 months of the experiment. How- ever, two tasks chosen to reflect shape discrimination or grouping abilities were severely impaired by the V2 lesions, again with no recovery evident over the 10 month survival period. Control observations made with related tasks suggested that the apparently selective loss was not due simply to task difficulty or to an inability to detect the elements of textures to be grouped. These results suggest that lesions of area V2 may selectively interfere with grouping processes without disrupting basic visual capacities.

\section{Effects of $V 1$ and $V 2$ lesions on basic capacities}

The V1 lesion in the current study entirely eliminated acuity and contrast sensitivity, a result consistent with many previous studies that have shown that V1 lesions abolish most visual capabilities (see below). This result is readily explained by the fact that virtually all of the geniculocortical projection passes through V1, so V1 lesions remove most of the input to visual cortex. For this reason, it is not possible to dissociate the contribution that V1 makes as the source of visual input for extrastriate cortex from any specific behavioral contributions that

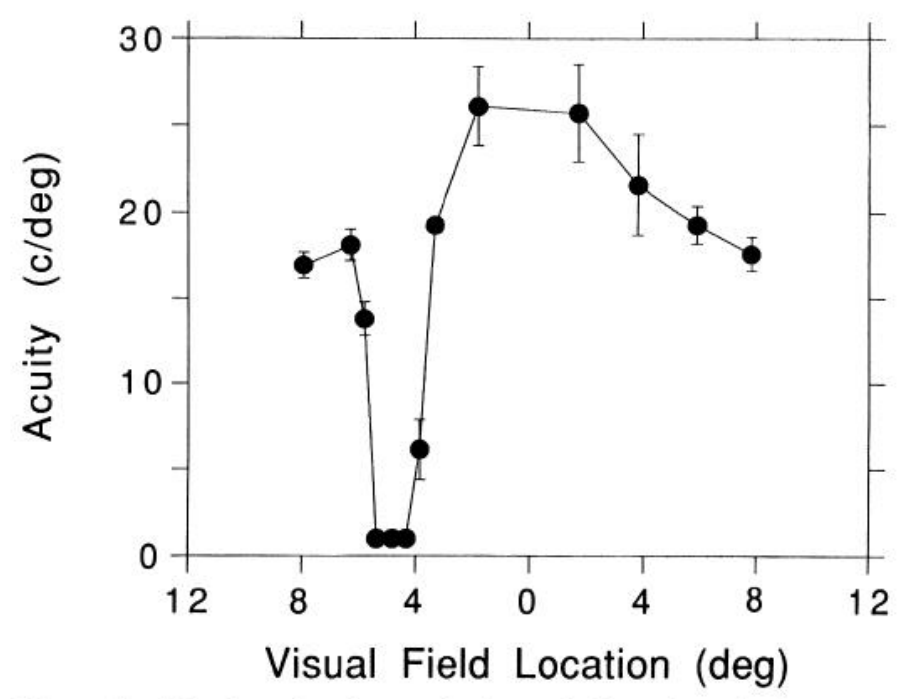

Figure 6. Visual acuity along a horizontal plane through the upper visual field of monkey 9102 . The plane is $2^{\circ}$ above the fovea. An abrupt decrease in acuity was found at about $4-6^{\circ}$ in the left upper field, corresponding to the location of the V1 lesion. Error bars are \pm SEM. 

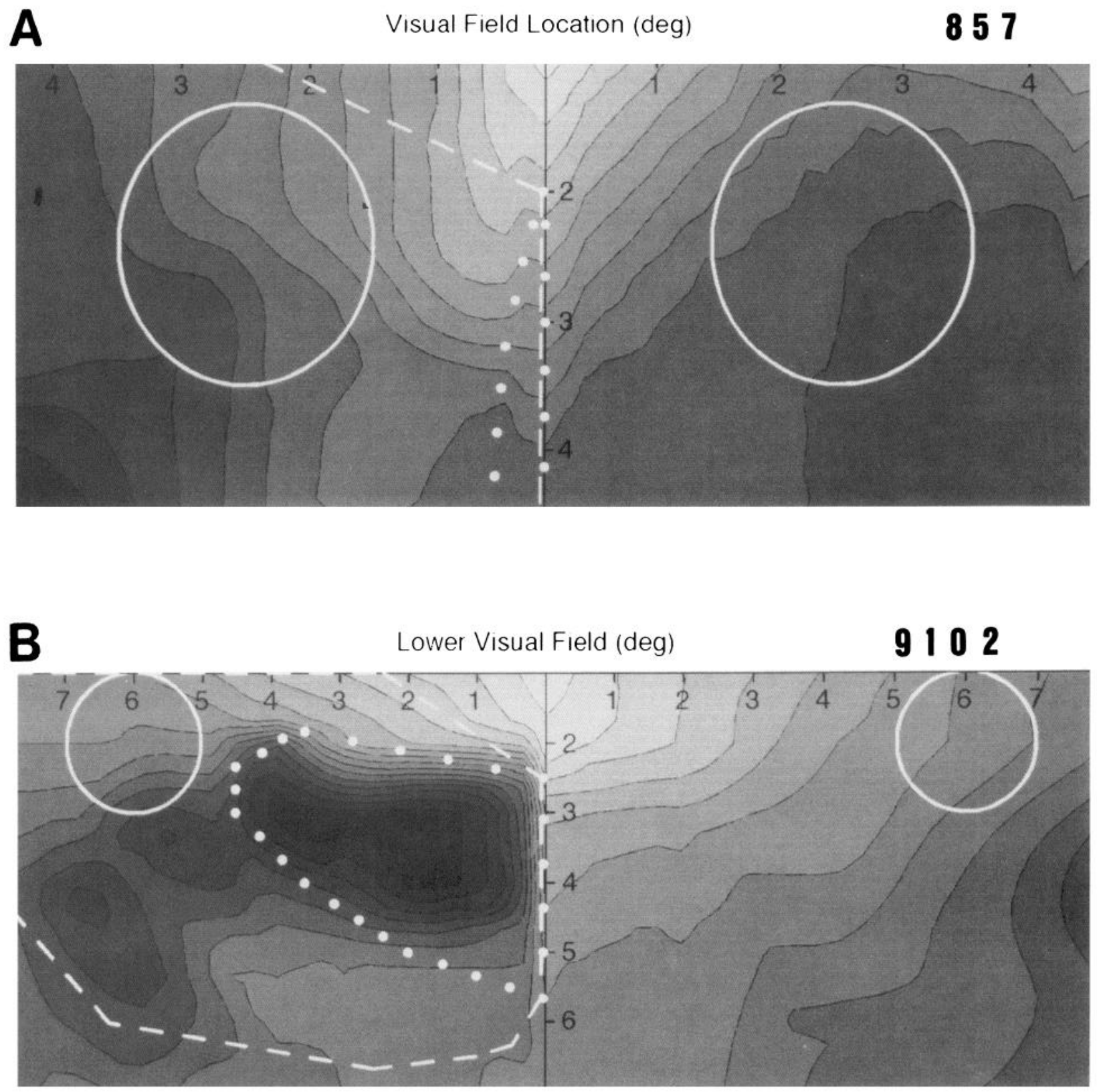

Figure 7. Visual acuity across the lower visual field of monkeys 857 and 9102 . Acuity was measured at $2^{\circ}$ intervals in monkey 857 , and with $1^{\circ}$ spacing in monkey 9102. The vertical axis corresponds to the vertical meridian of the visual field, and the horizontal axis is $1^{\circ}$ below the horizontal meridian. The highest acuity was at the origin of the axes, and it was approximately the same in monkey 857 and in monkey 9102 . Successive contour lines represent about a 10\% decrease from this acuity. Dotted lines show the location of inadvertent damage to cortical area V1, and dashed lines damage to area V2. The circles represent the location and approximate extent of the test locations for contrast sensitivity and orientation of lines of dots or texture elements.

it provides. V2 is the first, and largest, cortical visual area that can be lesioned without removing most of the input to later stages. Because V1 sends direct projections to V3, MT, and V4, ablation of $\mathrm{V} 2$ leaves some routes to higher visual cortex intact. The effects of the V2 lesions were dramatically different than those of V1 lesions, causing no change in acuity, and little or no change in contrast sensitivity.

The survival of acuity and contrast sensitivity after V2 lesions is consistent with the notion that these functions can be mediated without the involvement of extrastriate cortical visual areas. It is also possible that the other extrastriate projections from V1, to V3, MT, and V4, can support these functions.
Whether low-level visual functions require extrastriate visual cortex remains a basic and important question about how visual cortex works.

Despite the fairly complete sparing of basic psychophysical thresholds, we do not know if the appearance of the test stimuli was altered by the lesion. Since we used an orientation discrimination (Katz and Merigan, 1988; Pasternak and Olson, 1992) rather than a detection task (Miller et al., 1980) to test both acuity and contrast sensitivity, we know that the monkey could still discriminate orientation after the lesion. Other features of the stimulus, especially those contributed by area V2 or its projections, might have changed. One example of such an ap- 

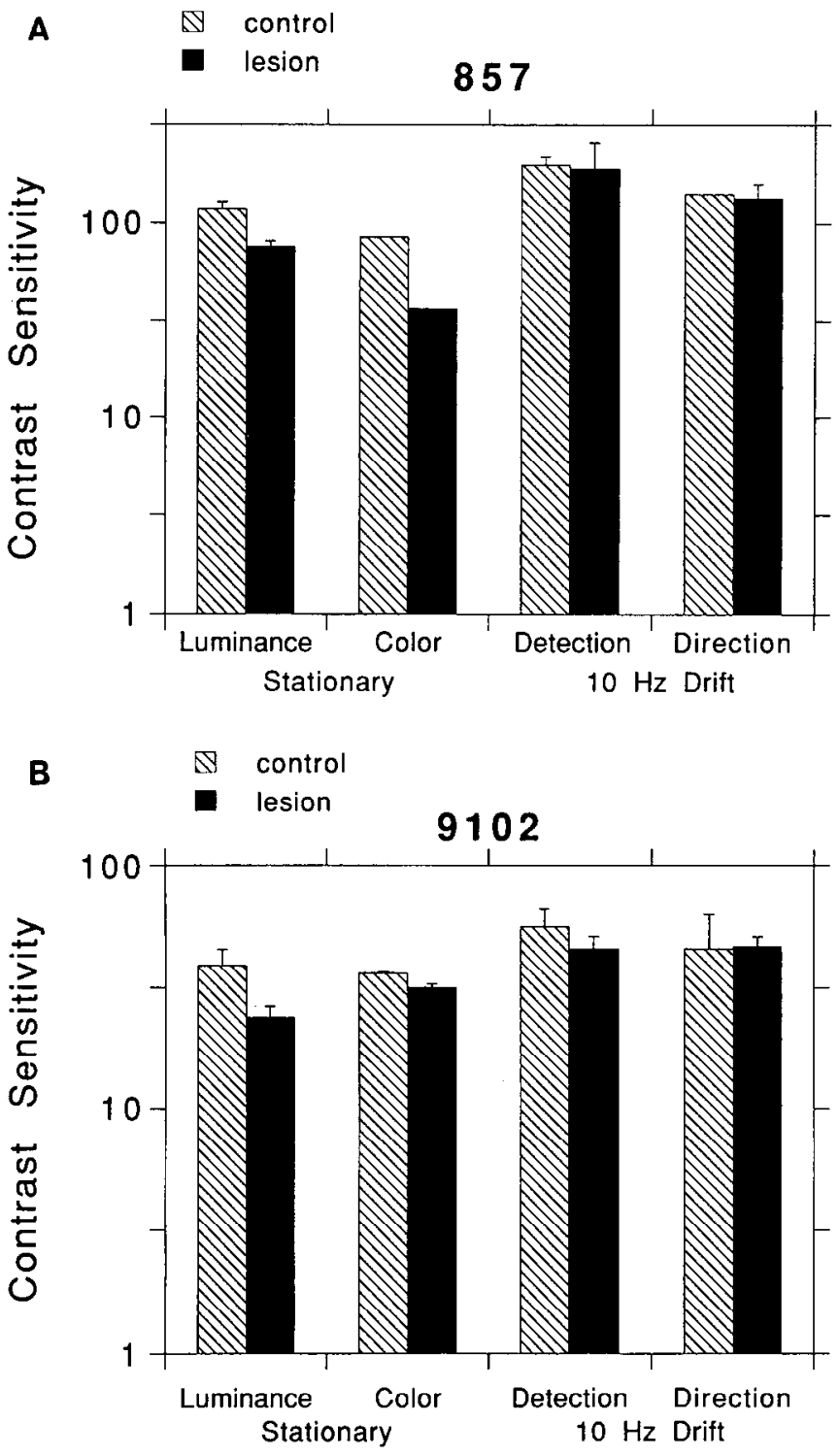

Figure 8. Contrast sensitivity of monkeys 857 and 9102 in control and V2 lesion locations (see Fig. 5 for visual field locus) for four types of Gabor stimuli. Luminance sensitivity was measured with stationary, 1 cycle/degree grating patches; Color sensitivity with stationary, 1 cycle/ degree isoluminant red-green grating patches; Detection with $10 \mathrm{~Hz}$, rightward drifting, I cycle/degree grating patches; and Direction with identical grating patches that drifted either right or left. The value shown for Color is chromatic contrast sensitivity (sum of the modulation of middle- and long-wavelength cones), and for the other tests is Michaelson contrast $\left(L_{\max }-L_{\min } / L_{\max }+L_{\min }\right)$. The only significant difference was color contrast sensitivity for monkey $857(t=22, \mathrm{df}=1, p>0.05)$. Error bars are \pm SEM.

pearance change has been reported when grating stimuli were made to exceed the Nyquist sampling frequency for human observers (Brainard et al., 1992). The grating appearance was so degraded by such presentation that, although their orientation could be discriminated, it was not possible to distinguish spatial frequency from contrast changes. We know from portions of the present study that, after V2 lesions, monkeys were not able to discriminate patterns on the basis of groups of details. It is possible that even more fundamental features of the visual stimuli, for example, spatial phase, uniformity, and so on, were also obscured by the lesions, and that such a change of appearance

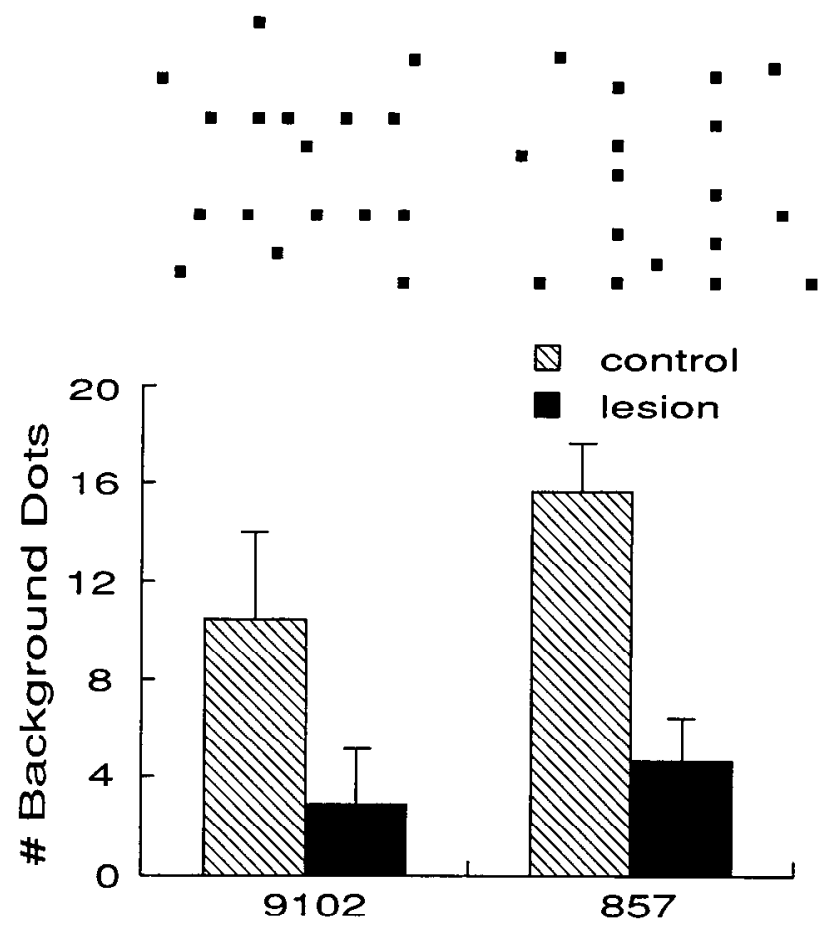

Figure 9. The number of background dots that brought the discrimination illustrated above the data to threshold performance. Results are shown for both monkeys in control and V2 lesion locations. On each trial only a single stimulus was presented. The stimulus shown to the left above the data has two horizontal lines of dots masked by seven background dots and indicated that a left response was correct. That to the right has two vertical lines of dots masked by seven background dots and indicates that a right response was correct. Stimuli indicating left and right responses are shown in the same way in Figures 10-13. Error bars are \pm SEM. The lesion effect was significant for monkey 857 $(t=15, \mathrm{df}=1, p>0.05)$.

might have caused the transitory disruption of acuity by $\mathrm{V} 2$ lesions that was seen in monkey 857 in this study.

The present failure to find dramatic changes in either acuity or contrast sensitivity contrasts sharply with the results of Horton and Hoyt (1991b), who found dense visual field loss below the horizontal meridian in human patients with cortical lesions. They felt that the sharp demarcation of the field loss along the horizontal meridian suggested that the source of the loss was damage to an extrastriate area, such as V2 or V3, that had a separated representation of the upper and lower visual field. Damage to area V1 was considered less likely to be the source of the loss because upper and lower visual field representations are contiguous in $\mathrm{V} 1$, and it would require an unusual lesion to produce such a sharp horizontal border of field loss. The present results suggest that V2 or V3 lesions are probably not the basis of this field loss if the human visual cortex is similar to that of the macaque. A more likely basis would be damage to extrastriate projections of the lower visual field coursing superiorly from area V1. Such a lesion would be quite different from that studied here, which appeared from myelin stains to damage only cortical neurons and not cortical pathways, and would therefore have left intact projections from area $V 1$ to all extrastriate areas except for V2 and portions of V3.

\section{Performance with colinear dots}

The detection of colinearity is thought to be an important basis for many visual grouping abilities (Lowe, 1985). Its presumed 


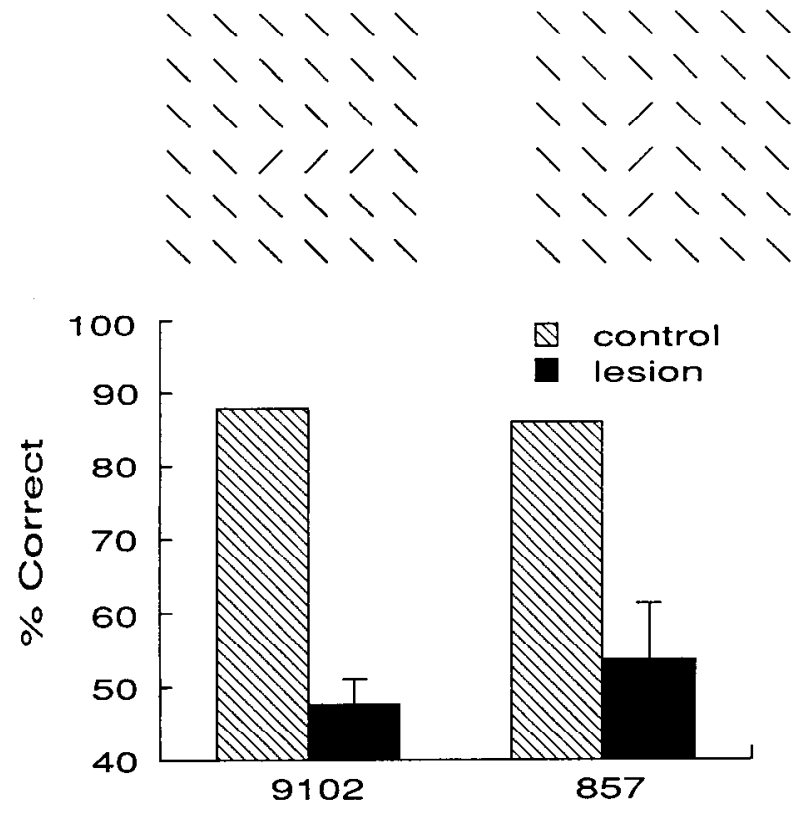

Figure 10. Percent correct performance for the two monkeys in control and V2 lesion locations for the discrimination task illustrated above the data. The stimulus to the right has a vertical row of right-oblique lines, and that to the left has a horizontal row. The monkey was required to identify the orientation of the row of differently oriented segments. Error bars are \pm SEM. Both lesion effects were significant $(t=21,23$; $\mathrm{df}=1 ; p>0.05$ ).

importance derives from the consideration that one task of the visual system is to determine the 3-D shape of ohjects from 2-D retinal images and that colinearity is one of the few features of 3-D images that survives projection onto a 2-D plane (Lowe, 1985). Thus, it is likely that the perception of colinearity is important to shape perception. It is not necessary to invoke complex grouping phenomena to account for orientation discrimination with colinear dots when they are presented with no background dots. Under these conditions, the task is similar to a vertical-horizontal discrimination of line or grating stimuli, it can presumably be mediated by any linear, orientation-tuned mechanism, and it is not disrupted by V2 lesions (see above). As background dots are added, the signal-to-noise ratio for the discrimination by a linear oriented mechanism is gradually reduced, until it becomes very unfavorable for large numbers of dots. It has been proposed that when there is high background masking, perceptual grouping processes, such as detection of colinearity, may aid discrimination (Lowe, 1985). According to this analysis, the present finding may suggest that the high level grouping response of collinearity detection has been disrupted by V2 lesions. An alternative account, which cannot be rejected by the present data, is that $\mathrm{V} 2$ lesions simply increase the masking effect of background dots.

\section{Discriminating the orientation of a group of texture element}

Texture segmentation can often be accomplished by linear grouping or filtering operators (Bergen and Adelson, 1988; Nothdurft, 1990), if the linear operator that does the segmentation responds to the difference between texture elements in the regions to be segregated. However, when the texture is so constructed that the operator involved in segmentation cannot see a difference between texture elements (e.g., Fig. 10), then a more complex nonlinear operation must be invoked to segment
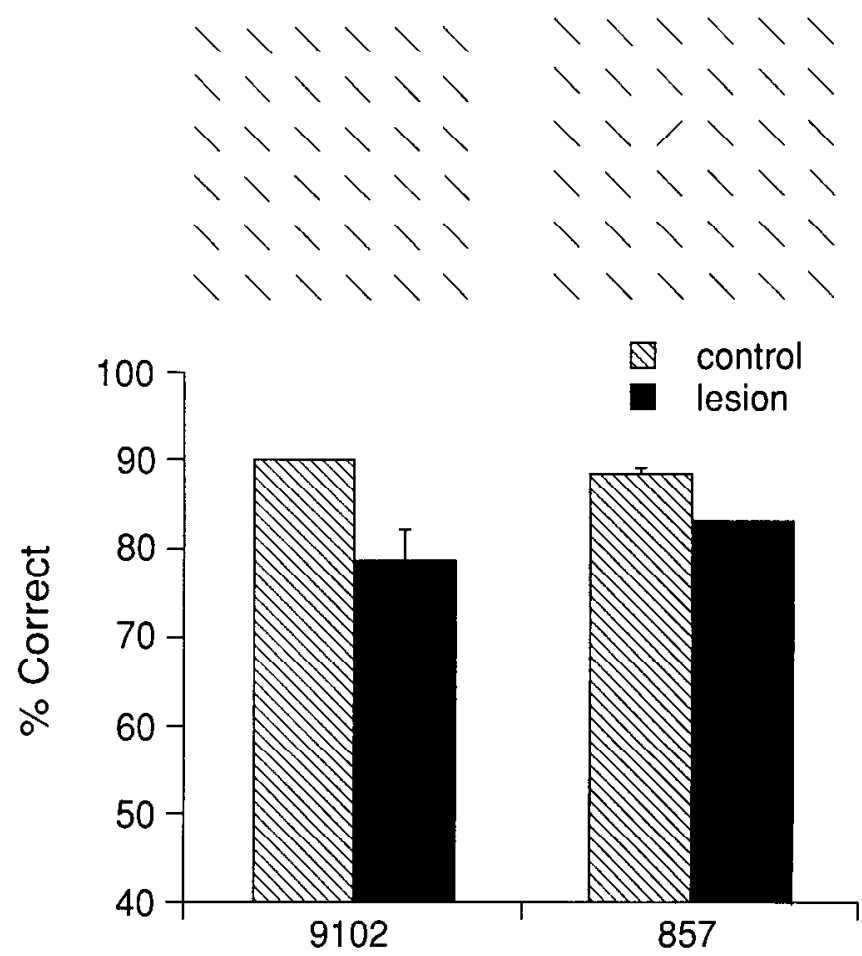

Figure 11. Percent correct performance on a discrimination used to determine if the monkey could detect differently oriented line segments. Performance is shown for both control and V2 lesion locations. Error bars are \pm SEM. The effect of the lesion was significant for monkey 857 $(t=11, \mathrm{df}=1, p>0.05)$.

the texture (Sutter et al., 1989). For the stimulus shown in Figure 10 , this could involve a local analysis of the orientation of each texture element followed by a rectifying stage or other process that preserves the sign of response, and an integrating stage that sums the rectified signal. Such processes have been termed second order or nonlinear (Bergen and Adelson, 1988), and the present experiment indicates that they are disrupted by V2 lesions.

Several control experiments were used to rule out other explanations for the observed deficit. A first possibility was that the local orientation analysis was disrupted by damage to area V2. The experiment illustrated in Figure 11 shows that local orientation contrast could be detected despite a V2 lesion. This suggests that the basis for the disrupted perception was not an interference with local orientation processing. A second possibility is that the global analysis of the orientation of the group of three texture elements was disrupted by the $\mathrm{V} 2$ lesion, perhaps masked by the presence of other texture elements. This account was contradicted by the second control condition (Fig. 12), in which the overall orientation discrimination could be made despite the surrounding texture, if a luminance cue was added to the segmented stimulus. This stimulus requires only a simple linear filtering operation to reveal the correct orientation. Finally, we wanted to determine if the improvement from the stimuli shown in Figure 10 to that shown in Figure 12 was confined to adding luminance cues, or if other visual stimuli that could be detected by linear filtering would also result in such an improvement. To examine this, we made the basis for the texture segmentation the color of the texture elements, and then checked that varying the intensity around a calculated isoluminance did not disrupt the discrimination. After the mon- 

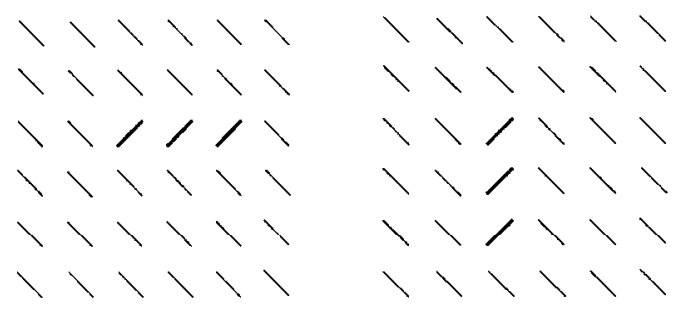

பั

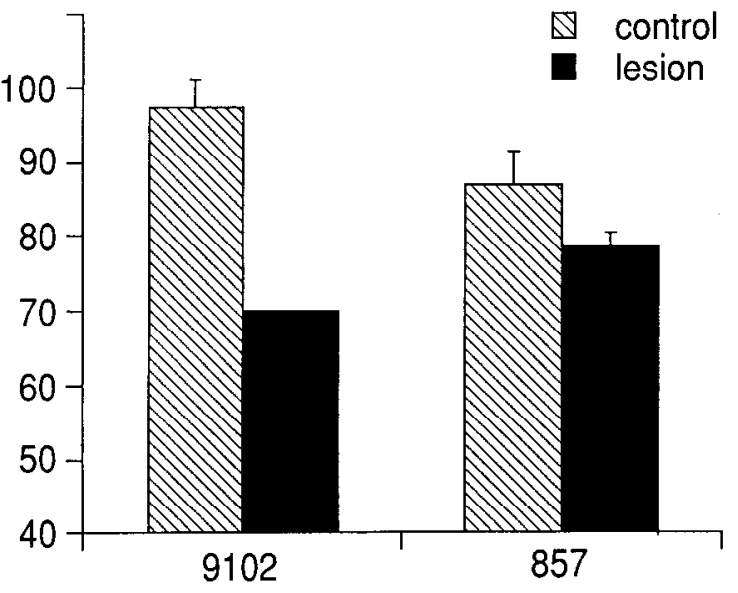

Figure 12. Percent correct performance on a discrimination task used to determine if the monkeys could perform the task illustrated in Figure 9 when the differently oriented line segments were made three times as thick. Error bars are \pm SEM. The lesion effect was significant for monkey $9102(t=22, \mathrm{df}=1, p>0.05)$.

keys reached stable performance on this discrimination with central vision, they were switched to peripheral viewing, and they performed very well on the task, both in control and in V2 lesion locations. Since this task and those shown in Figures 11 and 12 could be performed using simple linear filtering operations, we have tentatively concluded that only discriminations that require nonlinear visual processing are disrupted by V2 lesions.

The present result does not indicate a likely cortical locus for nonlinear visual analysis. It does seem that simple cells in area V1 (Hubel and Wiesel, 1968) have the appropriate properties to respond in an orientation-specific fashion to the stimuli shown in Figures 12 and 13, but that they could not respond appropriately to the stimulus shown in Figure 10. On the other hand, complex cells within area V1 do show the type of marked nonlinearities that may be needed to respond to the oriented texture shown in Figure 10. Computational models of complex visual discrimination (Sutter et al., 1989; Malik and Perona, 1990) typically do not identify stages in the model with particular cortical areas, bccause there is such a wide range of neuronal properties within a single cortical area. It is also difficult to determine if nonlinear responses seen in single neurons (Heydt and Peterhans, 1989; Grosof et al., 1992) represent ascending, descending, local, or multiple sources. The results of this study do not clarify this issue, but do show that damage to area V2 disrupts discriminations involving second-order processing. It is not known which neuronal circuits are involved in this disruption.

\section{Previous studies of VI lesions}

Damage to cortical area V1 in humans typically results in almost complete loss of visual function in the corresponding region of
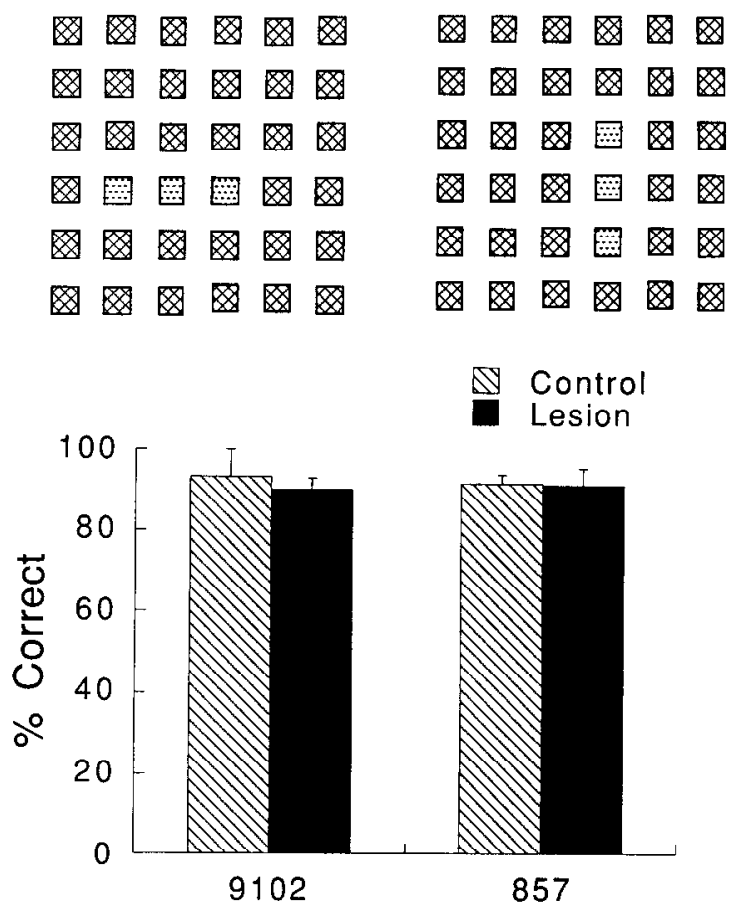

Figure 13. Percent correct performance on a discrimination task used to determine if a discrimination like that shown in Figure 10 could be done when the row of distinctive texture elements were of different color rather than different orientation. Error bars are \pm SEM. Lesions caused no significant effect.

the visual field (Koerner and Teuber, 1973). Such a profound loss is not surprising, given that virtually all geniculocortical fibers terminate in area VI (Shapley and Perry, 1986). This loss has been used to map coarsely the visual field correspondcncc of V1 in humans (Horton and Hoyt, 1991a), and is used routinely to localize the probable site of lesions in clinical neurology (Harrington, 1976). The complete loss of visual function we found in the visual field location corresponding to the V1 lesion in monkey 9102 is thus consistent with the traditional view of the role of area $\mathrm{V} 1$.

However, in recent years it has been noted that both humans and monkeys may show residual visual capacities in portions of the visual field that correspond to lesions of area V1 (Cowey and Stoerig, 1991). These unexpected abilities are typically ascribed to the use of visual pathways that avoid V1, such as collicular, pulvinar, or geniculoextrastriate projections (Cowey and Stoerig, 1991). In humans, the residual vision is quite peculiar in that it can be demonstrated with forced-choice techniques (e.g., "does it appear vertical or horizontal?"), but patients deny any consciousncss of the visual capacity. In monkeys, residual vision has been shown after apparently complete cortical lesions (Miller et al., 1980), although there remains some uncertainty about the precise qualities of this vision. In any case, the failure to uncover any evidence of residual vision after a V1 lesion in this study appears at odds with these reports.

The amount of time that has passed since the lesion could be important to recovery, given that some of the most dramatic evidence of "blindsight" in humans emerged only many years after occurrence of the lesion (Weiskrantz and Saunders, 1984; Cowey and Stoerig, 1991). It is not clear, however, that long postlesion times are necessary for some recovery of vision in monkeys. Detection of extremely intense spots of light has been 
found after 1 week in monkeys with either unilateral or bilateral V 1 lesions (Mohler and Wurtz, 1977). Reaching for and tracking of lights is seen within about 3 months after bilateral V1 lesions (Schilder et al., 1972; Dineen and Keating, 1981). A monkey tested 5 months after a bilateral lesion of striate cortex was able to detect and precisely localize moving targets or flashing lights (Humphrey and Weiskrantz, 1967). Thus, the 10 month survival time following the V1 lesion in the present study was undoubtedly sufficient to indicate that the lack of testable vision was due to some factor other than time.

In many of the studies demonstrating rapid recovery of some vision, the test stimulus was a bright light against a dark or dim background. The results of Mohler and Wurtz (1977) indicate that intensity is an important variable, since, shortly after a lesion, monkeys could only detect very intense spots. In other experiments, monkeys with VI lesions were able to discriminate lower-contrast stimuli more like those of the present study (Schilder et al., 1972; Dineen and Kcating, 1981), but only after extensive and progressive training. Furthermore, some monkeys failed to master the discriminations despite the lengthy training. This is clearly different from the present study, in which, although postlesion testing in and near the lesion locus was lengthy, we made no effort to retrain the monkey progressively through a series of tasks.

Another variable that distinguishes the present study from previous V1 lesion studies is the extent of the lesion. Subtotal V1 lesions have been used in some experiments (Mohler and Wurtz, 1977; Weiskrantz and Saunders, 1984) that have found rapid recovery of detection of intense punctate targets. However, those studies that have found recovery of more substantial vision (Schilder et al., 1972; Dineen and Keating, 1981; Weiskrantz and Saunders, 1984) have used complete V1 lesions either unilaterally or bilaterally. It is possible that recovery of function, no matter how extended or progressive the training, will not be substantial unless V1 lesions cover a large portion of the visual field.

The effects of V1 lesions have also been examined by determining their effects on the response properties of neurons in other cortical areas. Inactivation of V1 by cooling caused a near complete loss of response in V2 and V3 neurons, although about $30 \%$ of neurons in V 3 a continued to respond (Girard and Bullier, 1989; Girard et al., 1991a). The same investigators also found a complete loss of visual responses in area V4 when V1 was cooled (Girard et al., 1991b). These results are consistent with the notion that the studied cortical areas are strongly dependent on that major part of their input that comes from the geniculostriate pathway. However, a very different result was obtained for area MT. Rodman et al. $(1989,1990)$ found little change in the directionality, binocularity, receptive field size, or topography of macaque MT neurons after extensive Vl lesions, although the amplitude of responses was greatly reduced. The possibility that residual responses might reflect stimulation beyond the limits of the V1 lesion appears remote, given their subsequent finding that adding superior colliculus lesions eliminated residual MT responses. A similar sparing of MT neuronal response was obtained by Girard et al. (1992) with cooling of area V1. Again, response strength in MT was reduced, and directional tuning was broadened, but about $80 \%$ of recorded neurons remained visually active. A different result was obtained in an experiment done recently in the owl monkey (Kaas and Krubitzer, 1992), in which the response of MT neurons was recorded just before and just after ablating a portion of V1. This study found an abrupt loss of responsivity in MT neurons for stimuli placed in the affected region of the visual field, but no effect at other locations. These authors offered several possible accounts of why earlier studies might have obtained different results. Unfortunately, obvious differences between studies in variables such as species, anesthetic, and so on, make it difficult to compare results. However, if there is indeed a preservation of MT responses following V1 lesions, it is not clear what the role of such activity might be. If monkeys are blind within the portion of the visual field corresponding to $\mathrm{V} 1$ lesions, as the present study indicates, residual cortical responses are unlikely to be capable of mediating visual perception of the sort involved in the discriminations of this study. Future studies that compare V1 lesion effects on extrastriate responses and visual perception could clarify this issue.

\section{References}

Bergen JR, Adelson EH (1988) Early vision and texture perception. Nature 333:363-364.

Brainard DH, Williams DR, Sekiguchi N (1992) Supra-Nyquist resolution in the extra-fovea? Invest Ophthalmol Vis Sci 33:825.

Clarke S, Miklossy J (1990) Occipital cortex in man: organization of callosal connections, related myelo-cytoarchitecture, and putative boundaries of functional visual areas. J Comp Neurol 298:188-214.

Cowey A, Stoerig P (1991) The neurobiology of blindsight. Trends Neurosci 14:140-146.

Damasio H, Damasio AR (1989) Lesion analysis in neuropsychology. New York: Oxford UP.

Derrington AM, Krauskopf J, Lennic P (1984) Chromatic mechanisms in lateral geniculate nucleus of macaque. J Physiol (Lond) 357:241265.

Dineen J, Keating EG (1981) The primate visual system after removal of striate cortex. Exp Brain Res 41:338-345.

Finney DJ (1971) Probit analysis. Cambridge: Cambridge UP.

Foster KH, Gaska JP, Nagler M, Pollen DA (1985) Spatial and temporal frequency selectivity of neurones in visual cortical areas V1 and V2 of the macaque monkey. J Physiol (Lond) 365:331-363.

Girard P, Bullier J (1989) Visual activity in area V2 during reversible inactivation of area 17 in the macaque monkey. J Neurophysiol 62 : 1287-1302.

Girard P, Salin PA, Bullier J (1991a) Visual activity in areas V3a and $\mathrm{V} 3$ during reversible inactivation of area $\mathrm{V} 1$ in the macaque monkey. J Neurophysiol 66:1493-1503.

Girard P, Salin PA, Bullier J (1991b) Visual activity in macaque area $V 4$ depends on area 17 input. Neuroreport 2:81-84.

Girard P, Salin PA, Bullier J (1992) Response selectivity of neurons in area MT of the macaque monkey during reversible inactivation of area V1. J Neurophysiol 67:1437-1446.

Grosof DH, Shapley RM, Hawken MJ (1992) Monkey striate responses to anomalous contours. Invest Ophthalmol Vis Sci 33:1257.

Harrington DO (1976) The visual fields. St. Louis: Mosby.

Heydt R, Peterhans E (1989) Mechanisms of contour perception in monkey visual cortex. I. Lines of pattern discontinuity. J Neurosci 9:1731-1748.

Horton JC, Hoyt WF (1991a) The representation of the visual field in human striate cortex: a revision of the classic Holmes map. Arch Ophthalmol (Copenh) 109:816-824.

Horton JC, Hoyt WF (1991b) Quadrantic visual field defects. Brain 114:1703-1718.

Hubel DH, Wiesel TN (1968) Receptive fields and functional architecture of monkey striate cortex. J Physiol (Lond) 195:215-243.

Humphrey NK, Weiskrantz L (1967) Vision in monkeys after removal of the striate cortex. Nature 215:595-597.

Kaas JH, Krubitzer LA (1992) Area 17 lesions deactivate area MT in owl monkeys. Vis Neurosci 9:399-407.

Katz LM, Merigan WH (1990) Spatial resolution across the macaque retina. Vision Res 7:985-991.

Koerner F, Teuber H (1973) Visual field defects after missile injuries to the genicul-striate pathway in man. Exp Brain Res 18:88-113.

Lowe DG (1985) Perceptual organization and visual recognition. Norwell, MA: Kluwer. 
Malik J, Perona P (1990) Preattentive texture discrimination with early vision mechanisms. J Opt Soc Am 7:923-932.

Merigan WH (1989) Chromatic and achromatic vision of macaques: role of the $\mathbf{P}$ pathway. $J$ Neurosci 9:776-783.

Merigan WH, Katz LM, Maunsell JHR (1991a) The effects of parvocellular lateral geniculate lesions on the acuity and contrast sensitivity of macaque monkeys. J Neurosci 11:994-1001.

Merigan WH, Byrne C, Maunsell JHR (1991b) Does primate motion perception depend on the magnocellular pathway? J Neurosci 11: 3422-3429.

Miller M, Pasik P, Pasik T (1980) Extrageniculostriate vision in the monkey. VII. Contrast sensitivity functions. J Neurophysiol 43:1510 1526.

Mohler CW, Wurtz RH (1977) Role of striate cortex and superior colliculus in visual guidance of saccadic eye movements in monkeys. J Neurophysiol 40:74-94.

Nothdurft HC (1990) Texton segregation by associated differences in global and local luminance distribution. Proc R Soc Lond [Biol] 239 295-320.

Pasternak T, Olson CR (1992) Detection and discrimination performance after lesions of striate cortex in the cat. Invest Ophthalmol Vis Sci 33:1131.

Poggio GF, Gonzalez F, Krause F (1988) Stereoscopic mechanisms in monkey visual cortex: binocular correlation and disparity selectivity. J Neurosci 8:4531-4550.

Rodman HR, Gross CG, Albright TD (1989) Afferent basis of visual response properties in area MT of the macaque. I. Effects of striate cortex lesions. J Neurosci 9:2033-2050.

Rodman HR, Gross CG, Albright TD (1990) Afferent basis of visual response properties in area MT of the macaque. II. Effects of superior colliculus removal. J Neurosci 10:1154-1164.

Schilder P, Pasik P, Pasik T (1972) Extrageniculostriate vision in the monkey. III. Circle vs triangle and "red vs green" discrimination. Exp Brain Res 14:436-448.

Schiller PH, Malpeli JG (1977) Properties and tectal projections of monkey retinal ganglion cells. J Neurophysiol 40:428-445.

Shapley R, Perry VH (1986) Cat and monkey retinal ganglion cells and their visual functional roles. Trends Neurosci 9:229-235.

Sutter A, Beck J, Graham N (1989) Contrast and spatial variables in texture segregation: testing a simple spatial-frequency channels model. Percept Psychophys 46:312-332.

Van Essen DC (1985) Functional organization of primate visual cortex. In: Cerebral cortex, Vol 3 (Peters A, Jones EG, eds), pp 259-329. New York: Plenum.

Van Essen DC, Maunsell JHR (1980) Two-dimensional maps of the cerebral cortex. J Comp Neurol 191:255-281.

Weiskrantz L, Saunders RC (1984) Impairments of visual object transforms in monkeys. Brain 107:1033-1072.

Wong-Riley M (1979) Changes in the visual system of monocularly sutured or enucleated cats demonstrable with cytochrome-oxidase histochemistry. Brain Res 171:11-28. 